\title{
ADCNC-MAC: asynchronous duty cycle with network-coding MAC protocol for underwater acoustic sensor networks
}

\author{
Xiaoning Feng ${ }^{1^{*}}$, Zhuo Wang ${ }^{2 \dagger}$, Xiulong $\mathrm{Liu}^{1+}$ and Jiajie $\mathrm{Liu}^{1 \dagger}$
}

\begin{abstract}
To reduce the energy cost of underwater acoustic sensor networks (UWSNs), the duty cycle (i.e., periodic wake-up and sleep) concept has been used in several medium access control (MAC) protocols. Although these protocols are energy efficient, they sacrifice bandwidth utilization, which leads to lower transmission rate. In order to solve this problem, asynchronous duty cycle with network-coding Asynchronous Duty Cycle with Network-Coding MAC (ADCNC-MAC) is proposed. It contains initialization of the MAC protocol phase and data transmission phase. In the first phase, we use an asynchronous duty cycle to find a rendezvous time for exchanging data. A strategy to select network coder nodes is presented to confirm the number of network coder nodes and distribution in the network coder layer. In the data transmission phase, the network coder nodes transmit using the proposed network-coding-based algorithm and a higher volume of packet will be transmitted to the Sink with the same number of transmissions. Simulation results show that ADCNC-MAC achieves higher power efficiency, improves packet delivery ratio (PDR), and network throughput.
\end{abstract}

Keywords: Underwater acoustic sensor networks (UWSNs); Medium access control (MAC); Duty cycle; Network coding

\section{Introduction}

Underwater acoustic sensor networks (UWSNs) enable a wide range of application, including environment monitoring, tactical surveillance, disaster warning, and many more. UWSNs employ acoustic communications [1-5]. Compared with radio transmissions in wireless sensor networks (WSNs), the challenges of acoustic transmissions include (1) long propagation delay: The propagation speed for an acoustic link is $1500 \mathrm{~m} / \mathrm{s}$, $2 \times 10^{5}$ times lower than the speed of a radio link. This means the propagation delay is $2 \times 10^{5}$ times longer for an acoustic link. (2) Expensive transmitting power consumption: Power consumption for transmitting and receiving is similar in radio links. However, in acoustic links, the transmit power dominates and is typically 100 times more than the receive power. For example,

\footnotetext{
* Correspondence: fengxiaoning@hrbeu.edu.cn

${ }^{\dagger}$ Equal contributors

${ }^{1}$ College of Computer Science and Technology, Harbin Engineering University, Harbin 150001, China

Full list of author information is available at the end of the article
}

in the WHOI Micro-Modem [6], the transmit power is $10 \mathrm{~W}$ which is 125 times of the receive power $(80 \mathrm{~mW})$. (3) Lower available bandwidth: Influenced by harsh environment such as transmission loss, noise, and high propagation delay, the available bandwidth is limited and depends on both range and frequency.

Imperative and co-related research have shown energy as the most decisive resource for any UWSNs; the demand for extended network lifetime motivates the design of energy-efficient medium access control (MAC) protocols [7]. Consequently, the periodic wake-up and sleep schedule (namely duty cycle) MAC is introduced to reduce energy consumption. In this technique, each sensor node has its own wake-up schedule. For example, with a $10 \%$ duty cycle, a node has its state on only $10 \%$ of the time, resulting in substantial energy savings. In the active state, a node is able to transmit or receive data, but in the sleeping state, the node completely turns off to save energy [8].

\section{倠 Springer}

(c) 2015 Feng et al. Open Access This article is distributed under the terms of the Creative Commons Attribution 4.0 International License (http://creativecommons.org/licenses/by/4.0/), which permits unrestricted use, distribution, and reproduction in any medium, provided you give appropriate credit to the original author(s) and the source, provide a link to the Creative Commons license, and indicate if changes were made. 
So far, based on rendezvous selection between sender and receiver, duty cycle MAC protocols are mainly categorized into synchronous and asynchronous protocols. In a synchronous MAC [9], nodes in the same neighborhood are time-synchronized for simultaneous wake-up at the beginning of a common cycle and transmit their data. However, nodes' simultaneous wake-up in synchronous protocols results in higher collisions, and it is more difficult to implement in UWSNs.

Conversely, in asynchronous protocols [10], nodes maintain their individual cycles randomly and allow nodes to operate independently. Such protocols typically employ low power listening, in which, prior to data transmission, a sender transmits a preamble to rendezvous with the receiver [11]. When the receiver wakes up and detects the preamble, it stays awake to receive the data. These protocols achieve high energy efficiency and remove the synchronization overhead required in synchronous duty cycle approaches. However, duty cycle MAC protocols reduce bandwidth utilization and lead to a lower transmission rate.

The network-coding technique [12] improves the capacity of an information network with better utilization of bandwidth. In a multi-hop communication with network coding, the intermediate nodes of a network can appropriately encode the incoming data packets before forwarding the coded packets to the next node. The network-coding technique also improves reliability of the network. In this work, the selection of network coder node strategy has been proposed; we use an asynchronous duty cycle to find a rendezvous time for exchanging data in order to reduce energy consumption, and network coder nodes use a network-coding-based communication paradigm to improve the bandwidth utilization and reliability of the network.

The major contributions of this work can be summarized as follows: (1) According to the application of an autonomous underwater vehicle (AUV) in a fixed area to collect data, we defined the corresponding network model. (2) A selection strategy of network coder nodes is introduced, in order to confirm the number of network coder nodes and distribution. (3) Asynchronous schedule selection is proposed to confirm a rendezvous time for each sender-receiver pair. (4) A networkcoding-based algorithm is proposed to transmit data. (5) Simulations have been carried out to show the efficacy of the proposed approach in terms of network throughput, packet delivery ratio (PDR), and energy consumption.

The rest of this paper is organized as follows: Section 2 presents a background study on existing UWSNs of MAC protocols, Section 3 introduces detailed protocol operations, Section 4 presents performance evaluations by simulation efforts and comparisons, and Section 5 provides our conclusions.

\section{Related work}

The recent design of high transmission rate and energyefficient MAC protocols concentrated on terrestrial sensor networks [13] and the techniques that have been developed are not suitable for the challenging underwater acoustic communication medium that experiences a very large propagation delay of $1 \mathrm{~s}$ over $1.5 \mathrm{~km}$. In past acoustic network deployments, frequency-division multiple access (FDMA) was used, e.g., in the 1998-1999 SeaWeb [14] and in [15], but it was found to be restrictive and inefficient in terms of bandwidth utilization. SeaWeb 2000 favored a carrier sense multiple access/collision avoidance (CSMA/CA) solution with ready-to-send/clear-to-send (RTS/CTS) exchange; however, the problem with the application of handshaking protocols to UWSN is the energy consumption overhead caused by the RTS/CTS packets [14].

A recent underwater networking solution [16] proposed by Xie and Gibson seeks to achieve predictable end-to-end delays in the network by the use of a base station that computes routes for all underwater sensors. This obviates the RTS/CTS exchange and significantly reduces both the propagation delay and jitter along each route; however, there are issues regarding the scalability of this centralized solution to a large number of nodes.

The underwater acoustic network MAC (UWAN-MAC) [11] overcomes the energy consumption overhead problem with the "sleep mode" during transmission. In UWANMAC, SYNC beacons are transmitted by the sender; if the receiver detects the SYNC, it knows when to send data in the next cycle and then it goes to sleep. Hence, UWANMAC reduces the energy consumption through periodic wake-up. However, it lowers the transmission rate due to the duty cycle. Additionally, UWAN-MAC is a synchronous MAC protocol, it is difficult to synchronize among nodes in the network.

Over the years, several network-coding-based MAC protocols have been proposed for UWSNs. VBF-NC [17] is a reliable transport protocol for UWSNs. It transfers packets, coded by network coding, over relay node sets. However, error correction function of network coding cannot improve the throughput of the network and reduce transmission overhead. In order to solve this problem, the multi-path with network coding MAC (MPNC-MAC) [18] protocol is proposed by Shaobin Cai. In MPNC-MAC, three disjoint paths are established firstly, and then, two groups of packets $A$ and $B$, coded by network coding, are transmitted over the two side paths individually, and another group of packets $C(C=A \oplus B)$ are transmitted 
over the middle path. Hence, two groups of packets can be transmitted over three paths reliably. MPNCMAC provided higher throughput and reliability of data transmission. However, the MPNC-MAC protocol only applies to the sparse network, and it wastes energy consumption due to idle listening.

\section{ADCNC-MAC design}

\subsection{Network model}

In UWSNs, sensor nodes are randomly deployed in some fixed area to collect data, and we use AUVs or underwater gliders to receive data which the sensor nodes collected. According to the application above, we defined a UWSN network model as shown in Fig. 1.

The model is considered with $N$ sensor nodes scattered uniformly in an area, and all the $N$ sensor nodes are duty cycled. The nodes are named based on their roles in the network as shown in Fig. 1. The nodes are differentiated into three groups: relay sensor, network coder sensor, and the Sink. The relay sensor nodes generate data and transmit the generated data to the Sink. The network coder sensor nodes encode the raw native data which comes from the relay nodes before transmission. The Sink receive data and decode the data from network coder nodes. In the area, the relay nodes can communicate with the Sink using a multihop communication. However, the network coder nodes use a single hop to communicate with the Sink. The nodes in the range of the Sink are defined as the network-coding layer, and all the network coder nodes are in the layer.

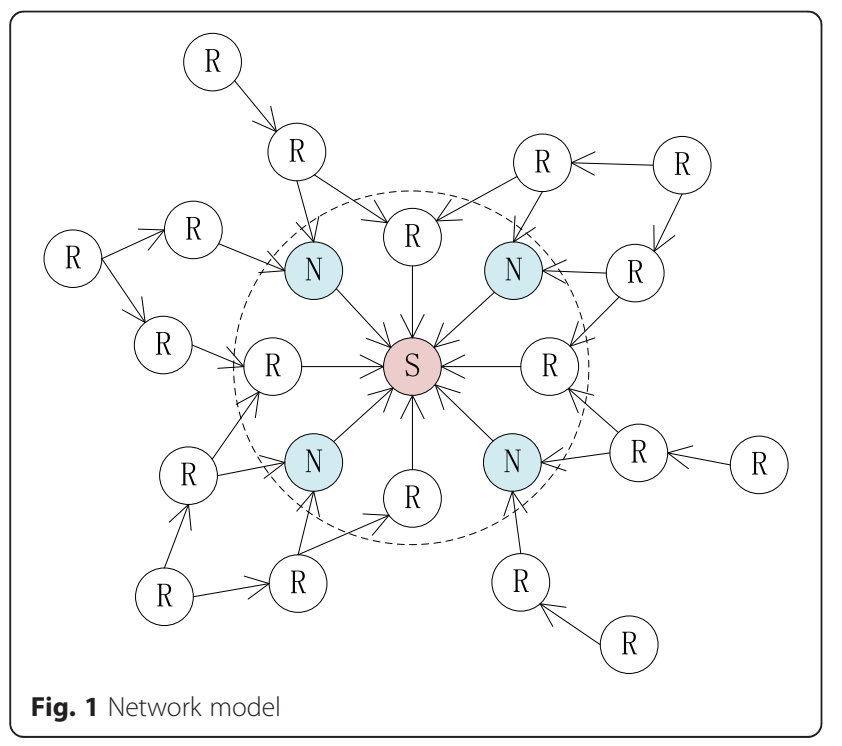

\subsection{Initialization of the MAC protocol}

The number of network coder nodes is an important parameter in the asynchronous duty cycle with networkcoding MAC (ADCNC-MAC) protocol; it will reduce the decoding rate of the Sink if the number of network coder nodes is too big. To ensure proper decoding of the network-coded packets at the Sink, not more than $50 \%$ of network coder nodes in the network-coding layer are needed [19]. Algorithm 1 represents the core operations of the selection of network coder nodes. $C_{n}$ represents the nodes in the network-coding layer, and $N_{n}$ is the number of nodes in the networkcoding layer. In the same way, $C_{s}$ designs the nodes that satisfy the conditions of the proposed algorithm, and $N_{s}$ is the number of nodes that satisfy the conditions.

The Sink broadcasts a selection of network coding node (SNC) packet to its neighbor nodes. The SNC packet contains the threshold of energy $\left(E_{n}\right)$ and the threshold of buffer $\left(B_{n}\right)$. For each neighbor node, it checks its own energy and buffer to determine whether it is larger than the threshold or not. Meanwhile, each node also checks its routing table $($ CheckRT ()$)$ to find if more than one node transmits data to it. The nodes will send acknowledgement (ACK) packets to the sink if they satisfy the above conditions. Each of the $A C K$ consists of its node's geographical position information [20].

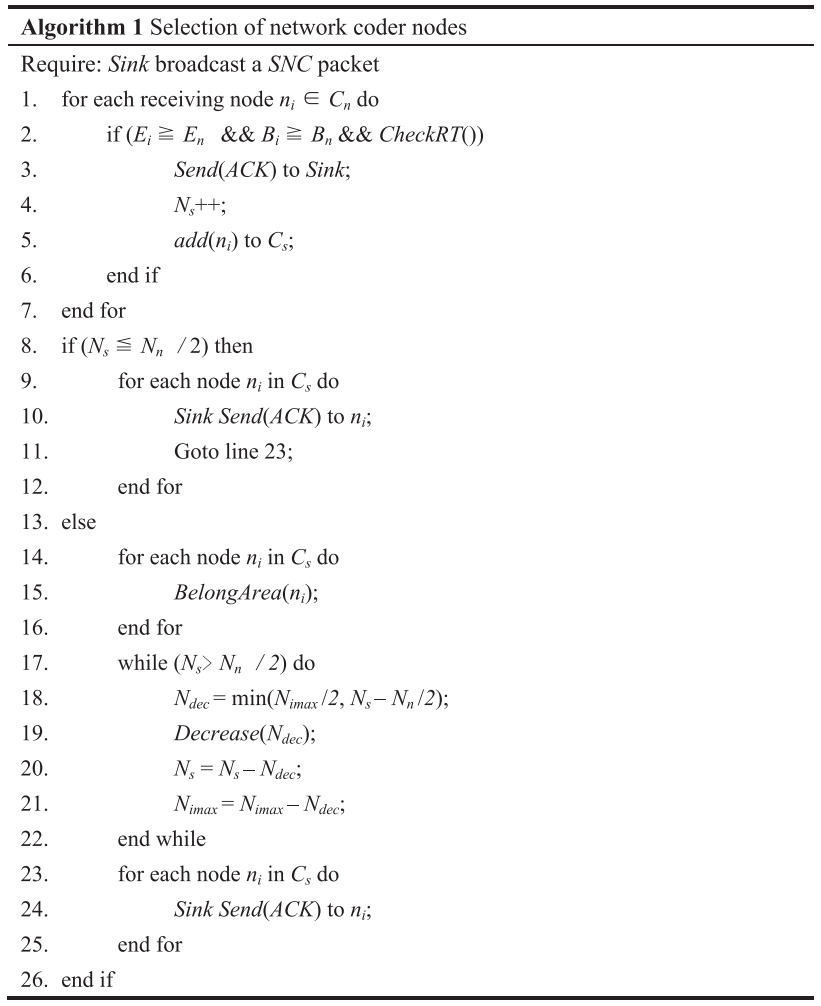


The Sink counts the received $A C K$ packets. If $N_{s} \leqq N_{n} / 2$, the sink confirms them as network coder nodes. If not, we divide the area of the Sink into $i$ parts equally. As the Sink knows each of the sent $A C K$ node's geographical position information, we can check the number of nodes in each area (BelongArea $\left(n_{i}\right)$ ). After that, we select the most nodes of area $\left(N_{\text {imax }}\right)$ to decrease in every cycle. We reduce $\min \left(N_{\text {imax }} / 2\right.$, $\left.N_{s}-N_{n} / 2\right)$ until $N_{s}=N_{n} / 2$. Then, the Sink announces the remaining nodes as network coder nodes. Finally, each node in the network-coding layer knows its role.

\subsubsection{Asynchronous schedule selection}

After the selection of the network coder node phase is complete, we could determine an asynchronous schedule for each sender-receiver pair. In Fig. 2, the direction of data transmission is $S \rightarrow R$. Node $S$ broadcasts a determine each of transmission (DET) packet (the shaded rectangle in the figure) at the beginning of its cycle period and then goes to sleep by turning off its transceiver circuits to save energy. This $D E T$ packet announces node $S$ s transmission cycle period " $T$." Assume that node $R$ is located near node $S$. The $R$ monitors the channel for DET packet. (The white rectangles in the figure indicate node $R$ 's receptions of node $S$ s $D E T$ packets.) After receiving the $D E T$ packet, node $R$ decodes the length of the transmission cycle period " $T$ " from this message then $R$ knows when to wake up to monitor $S$ after this period. In this method, all nodes can initialize their transmission/listen schedules.

We use the network in Fig. 3 as an example network configuration throughout this paper. In this figure, each node sets its transmission range and each circle indicates the transmission range of the node at its center. For this given network topology, the broadcasting of $D E T$ packets in the initialization period is illustrated in Fig. 4. In this figure, each node broadcasts its $D E T$ packet and remains awake until the beginning of the next cycle to receive its neighbors' $D E T$ packets. Node $S$ broadcasts its $D E T$ packet first. Then, node $S$ s neighbors receive the $D E T$ packet during initialization which allows them to schedule

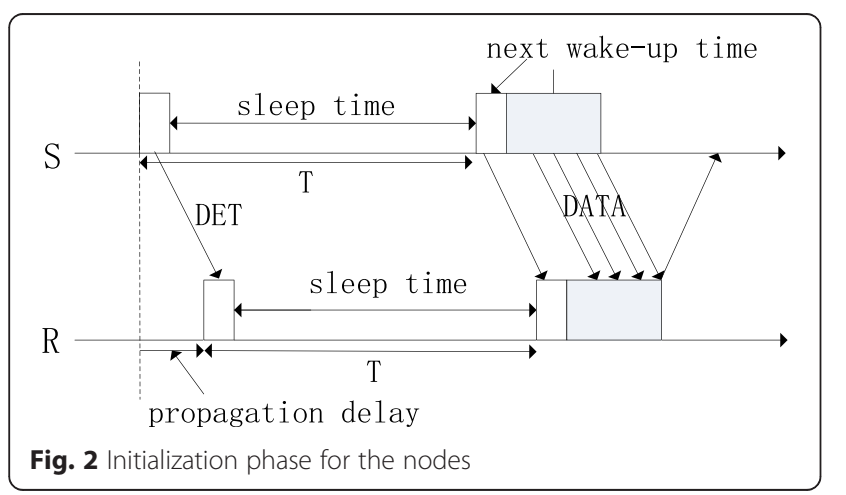

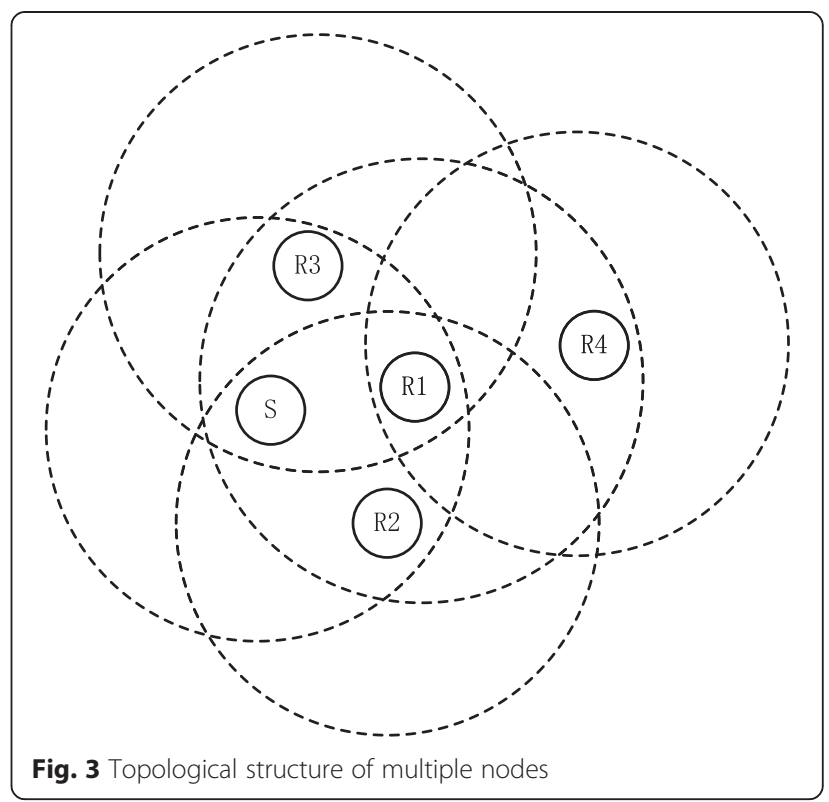

their wake-up times. Node $S$ will also schedule particular wake-up times as shown in this figure for its neighbor nodes $R 1, R 2$, and $R 3$ after it hears their schedules during this initialization period.

\subsection{Data transmission after initialization 3.3.1 Definition of packet structure}

After the initialization phase, each node has its role in the network model and knows when to wake up again to receive data from its neighbors. Starting with the next cycle, nodes follow their established schedules in the initialization period and begin sending data. Figure 5 shows the structure of the data transmission packet. The transmission duration has two distinct parts: "CF" and "DATA $T_{x}$." The "DATA $T_{x}$ " corresponds to the part where actual data is being sent. " $C F$ " is a control frame; it contains each node's cycle period " $T_{x}$ " which may be different from the one $(T)$ in the initialization period.

Figure 6 shows that during the data transmission phase, $S$ has the option to change its current cycle period by using the $C F$ message. Its neighbor $R$ can decode the modified $C F$ message and change wake-up times for that node, starting with the next cycle. It can avoid a time-consuming re-initialization by keeping a $C F$ message in the packet.

\subsubsection{Data transmission of nodes}

Whenever a node wakes up in the network-coding layer and receives a packet, it checks its role (refer Fig. 7). If it is a relay sensor node, it simply forwards the received data, whereas, network coder nodes follow the 


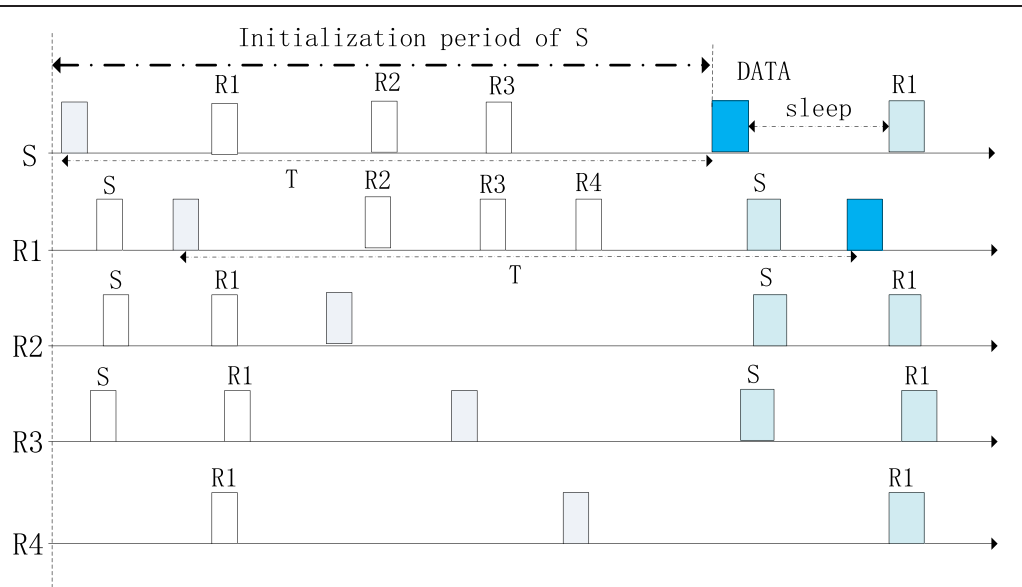

Fig. 4 Asynchronous duty cycle of multiple nodes

Algorithm 2 to process packets. Each node in the network-coding layer maintains a received queue $($ recQueue ()$)$ and a sensed queue (senQueue ()$)$. On receiving a packet $P_{i}$, a node puts the packet in $\operatorname{recQueue}\left(P_{i}\right)$. If the packet is already processed by the node, then it is discarded, otherwise checks senQueue() to determine whether it is empty or not. If it is not empty, pick a packet $P_{j}$ from senQueue() and coded with $P_{i}$, otherwise pick next packet $P_{i+1}$ from recQueue() and coded with $P_{i}$. On successfully creating an encoding packet, the node transmits the coded packet to the Sink. The processed packet is inserted into the forwarding set ForwardSet() which stores the forwarded packets. This helps in restricting further redundant transmissions.

Decoding of packets at the Sink: The Sink node receives native packets from the simple relay nodes and coded packets from the network coder nodes. In COPE [19], the intermediate nodes encode and decode packets. Unlike COPE, the decoding procedure is performed only at the Sink which processes all the gathered data in UWSNs. The Sink maintains a pool of packets, in which it stores each received native packet. When the Sink receives an encoded packet consisting of two native packets, the Sink retrieves the corresponding native packets one by one from

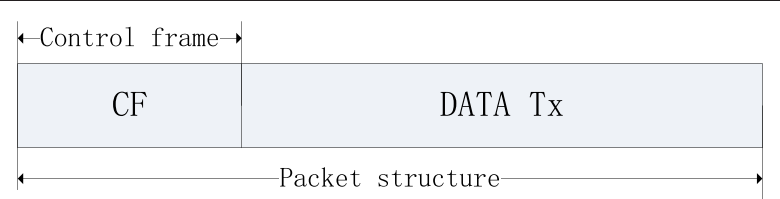

Fig. 5 Transmission packet structure the pool of packets. The Sink XORs the two native packets with the received coded packet to retrieve the missing packet which is totally lost or received with error at the Sink.

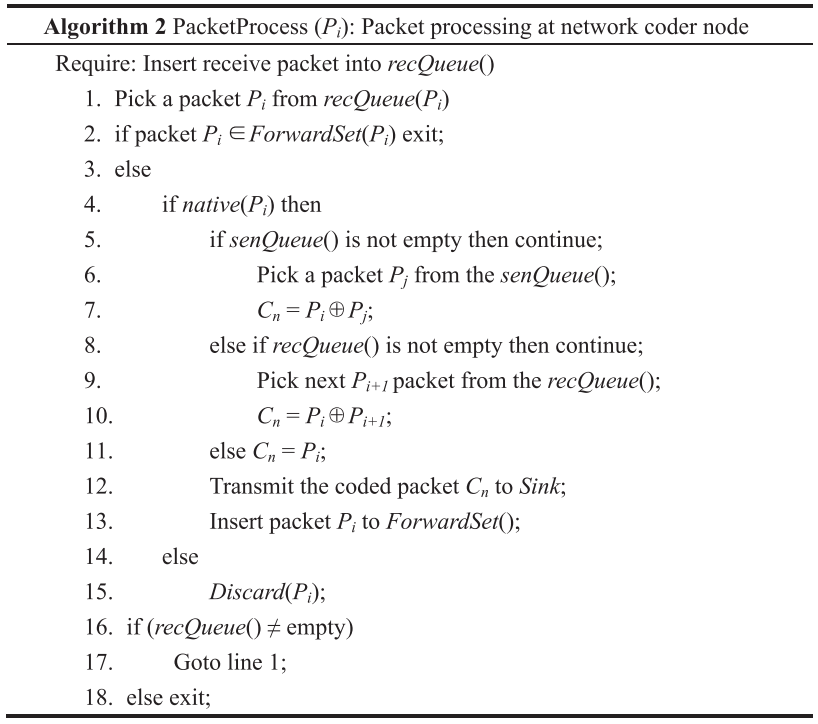

The above is best illustrated with an example in Fig. 8 . In Fig. 8a, network-coding node $N$ has three packets in its recQueue() $P_{1}, P_{2}$, and $P_{3}$. Relay node $R$ has one packet in its putout queue $P_{1}$. The table in Fig. $8 \mathrm{~b}$ shows the next hop of each node. Nodes $N$ and $R$ transmit packets to $S$. In Fig. 8c, node $N$ follows the Algorithm 2 to encode packets. It can process two coded packets $C_{1}=P_{1} \oplus P_{2}$ and $C_{2}=P_{2} \oplus P_{3}$. Node $R$ relays $P_{1}$ to the $S$. Node $S$ 


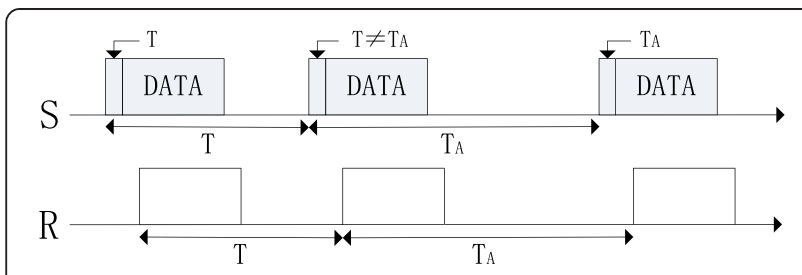

Fig. 6 Modification of the cycle period during the data transmission phase

decodes $P_{2}$ and $P_{3}$ in a single transmission. The above example shows that when packet $P_{2}$ or $P_{3}$ has an error, node $S$ can recover from network-coding packets.

\section{Performance evaluations}

The simulation of ADCNC-MAC is extensively performed in ns-3 [21], the performance of ADCNCMAC is analyzed in the $5 \mathrm{~km} \times 5 \mathrm{~km} \times 5 \mathrm{~km}$ area, and all senor nodes are randomly deployed in it. Experiments are done for 10 times in each scenario to acquire the average value as results. Table 1 provides the details concerning the ADCNC-MAC simulation parameters; the majority of them have been extracted from the ns-3 of the uan module (underwater acoustic network) [21]. This section presents two parts; the first part presents performance analysis of the proposed algorithm, and the second part presents performance analysis of ADCNC-MAC. To gather knowledge on the ADCNC-MAC performance, UWAN-MAC and MPNC-MAC protocols are implemented in the same simulation environment and compared with it.

\subsection{Performance analysis of the proposed algorithm} In order to demonstrate the effectiveness of the selection of the network coder node algorithm, we use three kinds of cases to compare. All of the cases are based on ADCNC-MAC, and only the number of network coder nodes in the network-coding layer is different. As we observe in Fig. 9, case 1 represents using the selection of network coder node algorithm. Case 2 represents no network coder nodes in the network-coding layer, and case 3 represents that all nodes in the network-coding layer are network coder nodes.

Figure 9a shows the relationship between the source data rate and throughput in different cases. It shows that the throughput performance of case 1 is better than case 2 and case 3 , since case 1 uses the proposed algorithm which improves the Sink's decoding rate and then improves the throughput. Case 3 leads that the Sink cannot decode when data transmits and reduces

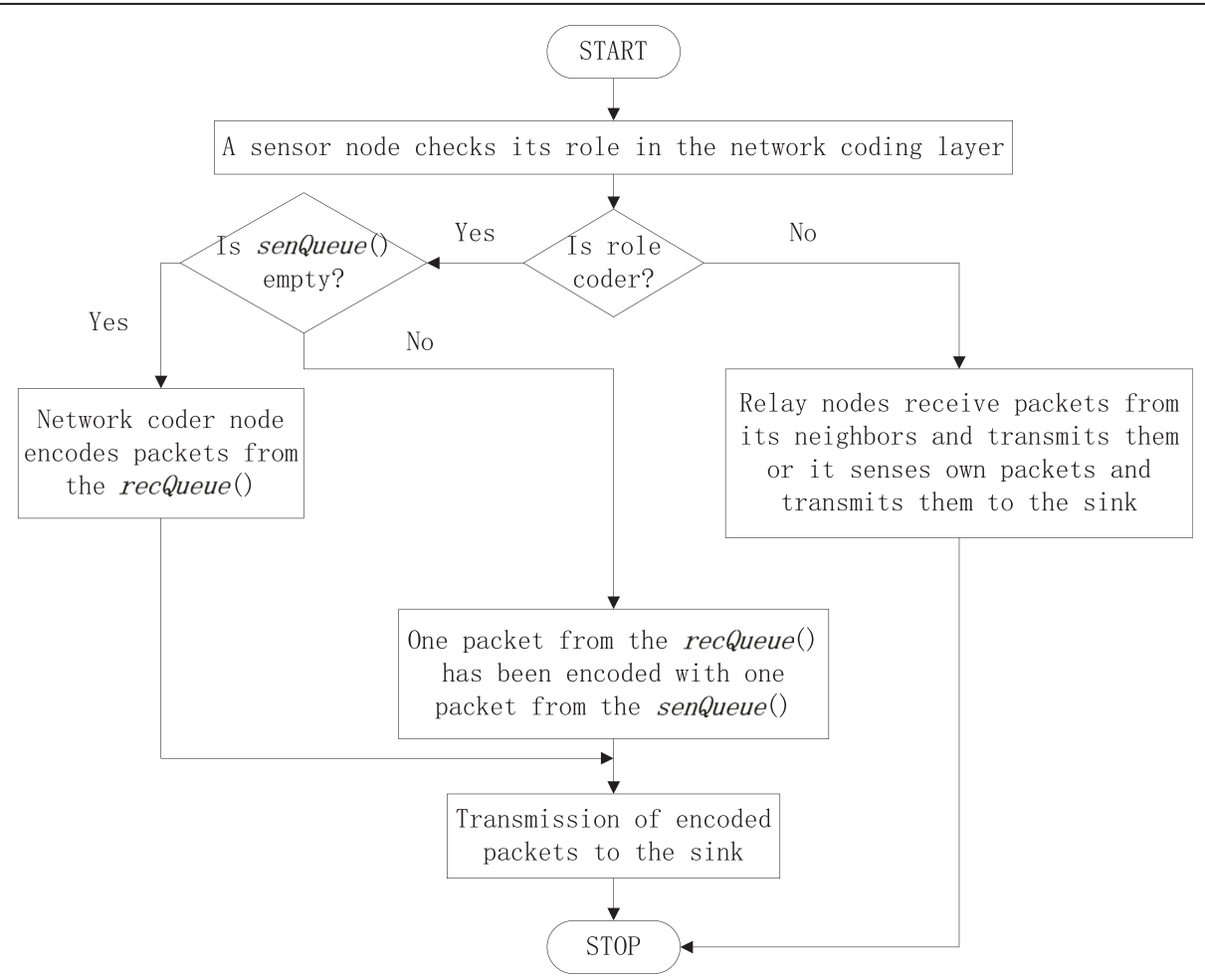

Fig. 7 Functionalities of the sensor nodes in the network-coding layer 


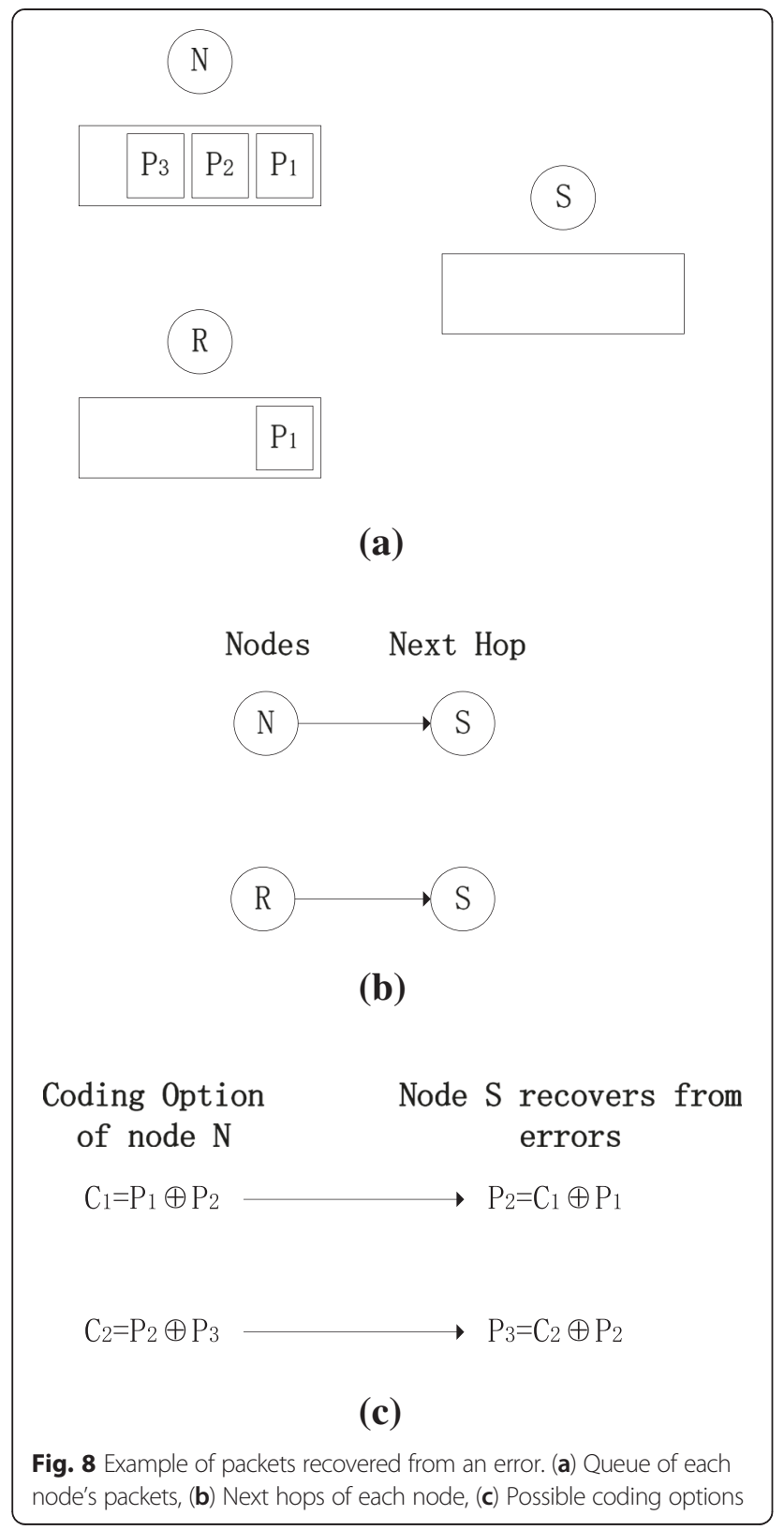

the throughput of the network. Case 2 has no network coder nodes in the network-coding layer; its performance is better than Case 3 .

Figure 9b shows the relationship between the source data rate and PDR in the different cases. It shows that the throughput performance of case 1 is better than case 2 and case 3; since case 1 has the highest decoding rate of the Sink, it can improve reception of data packets at the Sink. As source data rate increases, more and more collisions lead to lower PDR of the three cases. From Fig. 9, it can be seen that the proposed selection algorithm is strong
Table 1 Simulation parameters

\begin{tabular}{|c|c|}
\hline Parameter name & Parameter value \\
\hline Routing & Flooding \\
\hline Source rate & variable pps \\
\hline Packet size & 256 bytes \\
\hline Bandwidth & 80 bps \\
\hline Transmission range & $2000 \mathrm{~m}$ \\
\hline Duty cycle per node & 0.15 \\
\hline Error rate & 0.5 bps \\
\hline Initial energy/node & $10,000 \mathrm{~W}$ \\
\hline Transmit per packet & $50 \mathrm{~W}$ \\
\hline Reception per packet & $158 \mathrm{~mW}$ \\
\hline Idle & $158 \mathrm{~mW}$ \\
\hline Sleep & $5.8 \mathrm{~mW}$ \\
\hline Data rate & $160 \mathrm{bps}$ \\
\hline Underwater propagation model & Thorp Propagation Model \\
\hline
\end{tabular}

enough most of the time resulting in improved PDR and throughput.

\subsection{Performance analysis of ADCNC-MAC}

Figure 10 individually describes in different nodes the density, the relationship between the successful PDR, and the source data rate of protocols. Figure 10a shows when the nodes' density is in $0.25 /(\mathrm{km})^{3} \sim 0.5 /(\mathrm{km})^{3}$, UWAN-MAC gets the lowest performance as it is not using the network-coding technique. MPNC-MAC gets the best PDR when the source data rate is less than $0.05 \mathrm{pkt} / \mathrm{s}$. However, ADCNC-MAC gets better than MPNC-MAC when the source data rate is more than $0.05 \mathrm{pkt} / \mathrm{s}$. As MPNC-MAC sends data in three disjoint paths, more data collisions lead to lower PDR as source data rate increases. Figure 10b shows when the nodes' density is more than $0.5 /(\mathrm{km})^{3}$, ADCNC-MAC gets the best performance as the nodes' density increases, while MPNC-MAC gets lower PDR due to data collisions.

Figure 11 shows in different nodes the density, the relationship between throughput, and the source data rate of protocols. It can be seen that UWANMAC gets the lowest throughput as it is not using network coding. While the source data rate is less than $0.055 \mathrm{pkt} / \mathrm{s}$, MPNC-MAC is better than ADCNC-MAC. However, ADCNC-MAC is better than MPNC-MAC when the source data rate is more than $0.055 \mathrm{pkt} / \mathrm{s}$. As the source data rate increases, more data collisions lead to MPNC-MAC having a lower throughput.

In Fig. 12, MPNC-MAC suffers greater energy consumption due to idle listening and coding packets of 


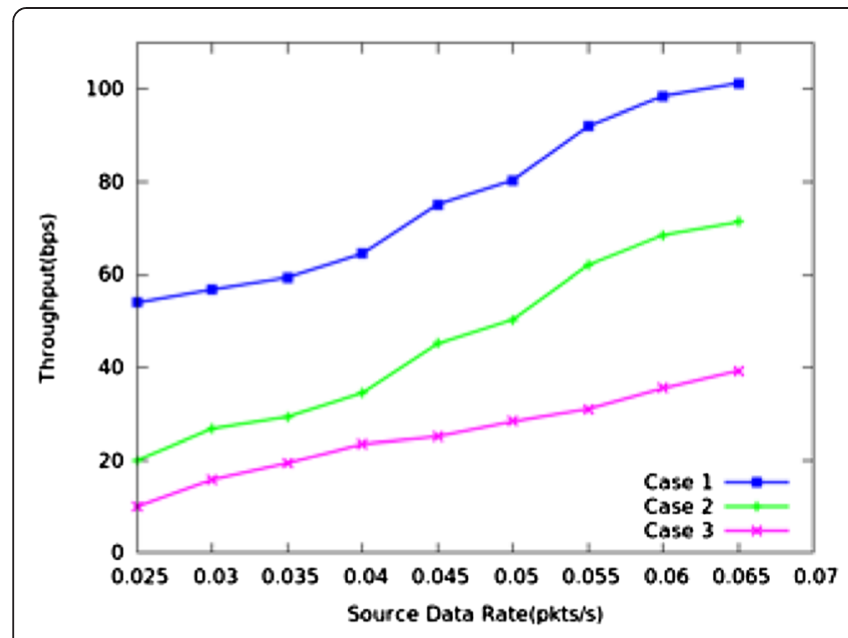

(a)

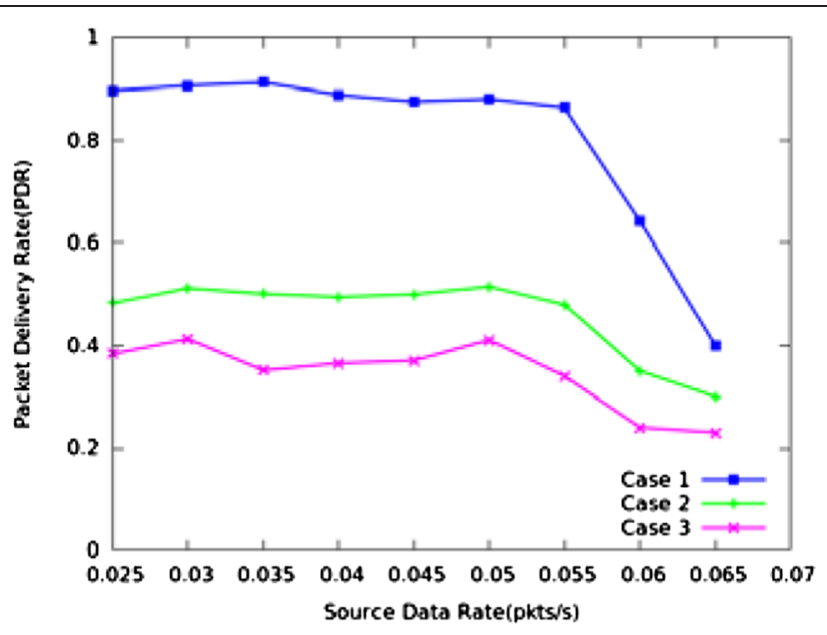

(b)

Fig. 9 Using selection of network coder node algorithm or not. (a) Relationship between the source data rate and throughput in different cases, (b) Relationship between the source data rate and PDR in different cases.

all nodes. ADCNC-MAC and UWAN-MAC maintain low energy consumption at a low rate due to using a duty cycle technique to save energy consumption. However, ADCNC-MAC has lower energy consumption than UWAN-MAC as network coding can fuse packets. It can reduce the number of sending packets and save more energy. As the source data rate increases, the three protocols of the energy consumption also increase for idle listening and collision-related retransmission. From Figs. 10, 11, and 12, the performance of ADCNC-MAC is better than that of MPNC-MAC and UWAN-MAC as a whole.

\section{Conclusions}

In this paper, we have proposed an innovative asynchronously scheduled duty cycle with network-coding MAC protocol for UWSNs. Unlike previous MAC protocols, ADCNC-MAC considers energy consumption, throughput, and PDR. In ADCNC-MAC, it uses a selection of network coder node algorithm to select proper

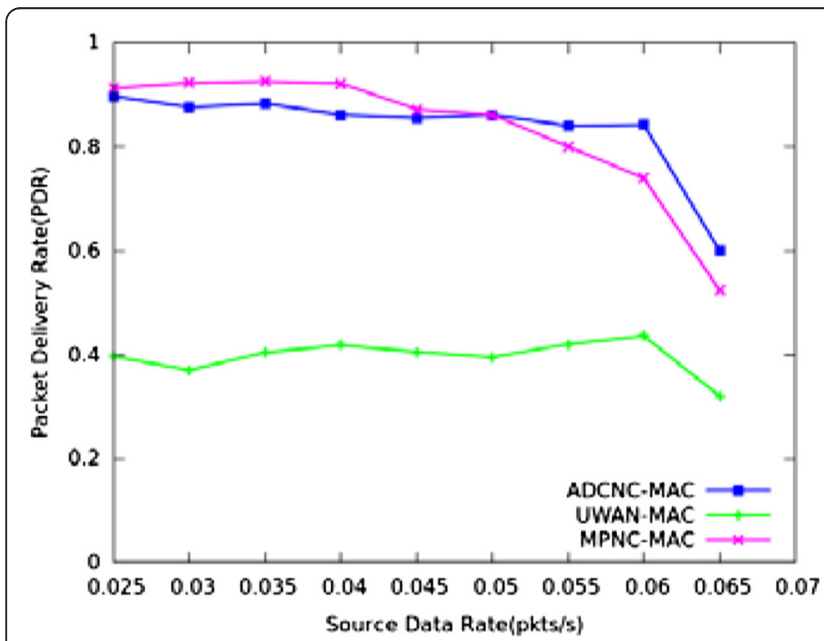

(a)

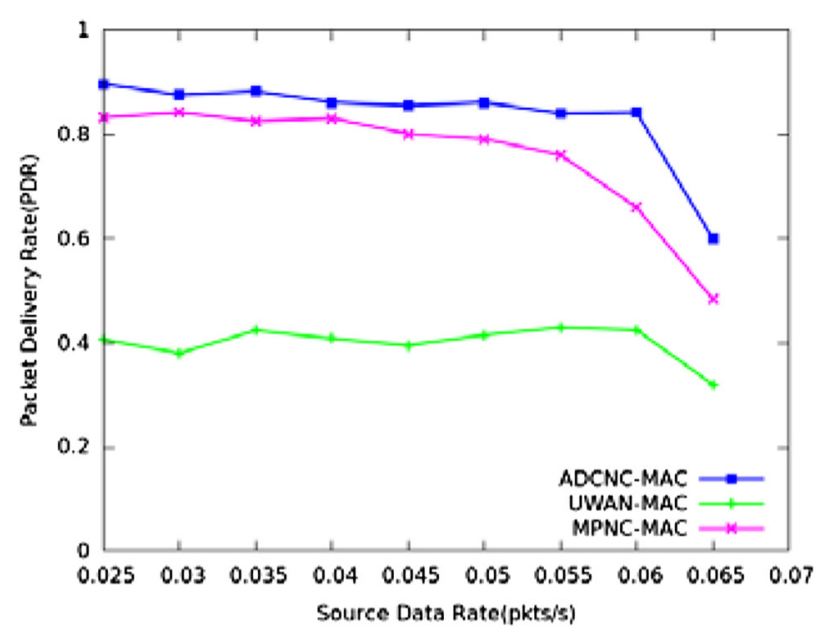

(b)

Fig. 10 The relationship between PDR and source data rate of protocols. (a) The nodes density in 0.25/(km)3 0.5/(km) ${ }^{3}$, (b) The nodes density more than $0.5 /(\mathrm{km})^{3}$ 


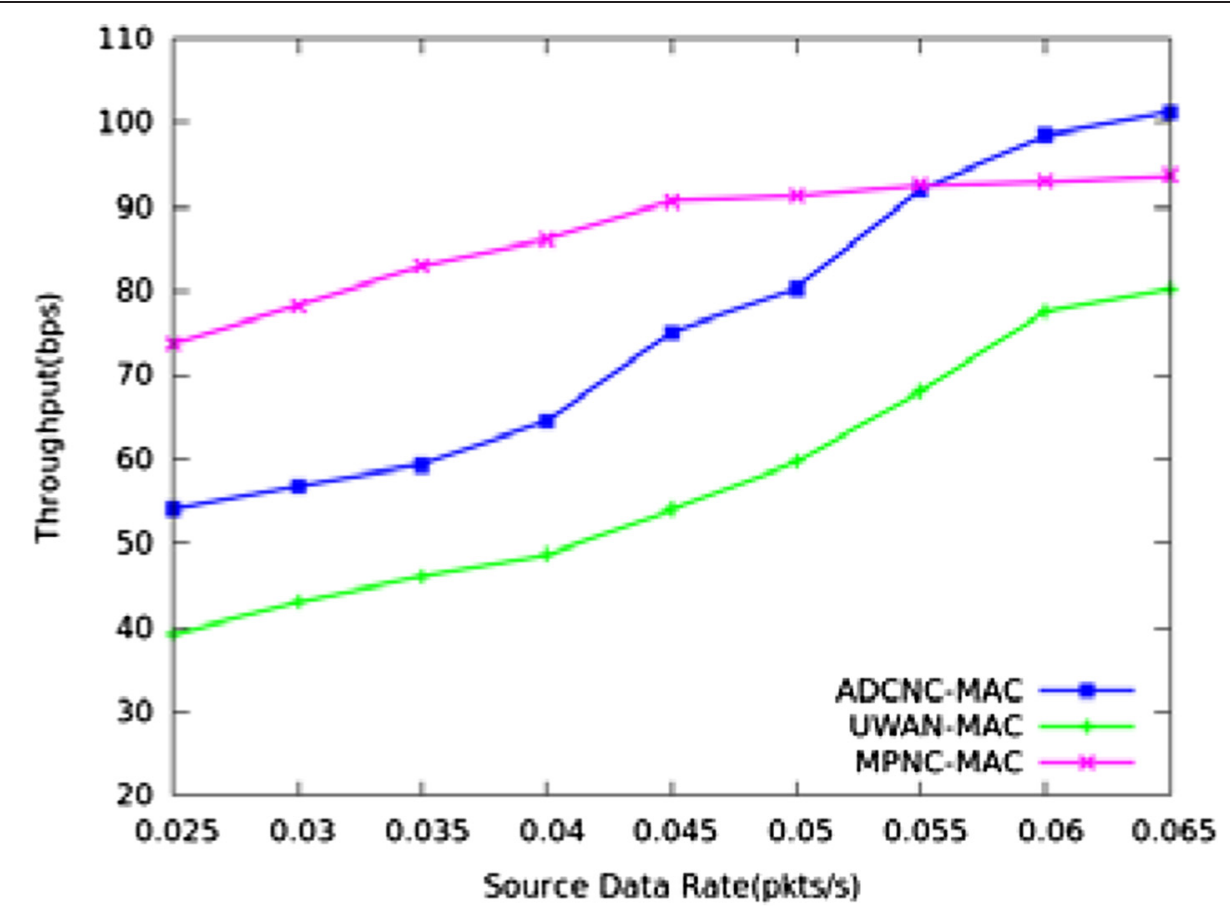

Fig. 11 The relationship between throughput and source data rate of protocols

network coder nodes before data transmission. In the data transmission phase, network coder nodes use a network-coding-based algorithm to code packets before transmitting them. This can make the Sink receive approximately $50 \%$ more data. Hence, it can significantly improve the PDR and throughput. Meanwhile, ADCNC-
MAC using an asynchronously scheduled duty cycle achieves a better energy consumption performance. The simulation results show that compared with MPNCMAC and UWAN-MAC, ADCNC-MAC achieves relatively lower energy consumption with the higher reliability of data transmission.

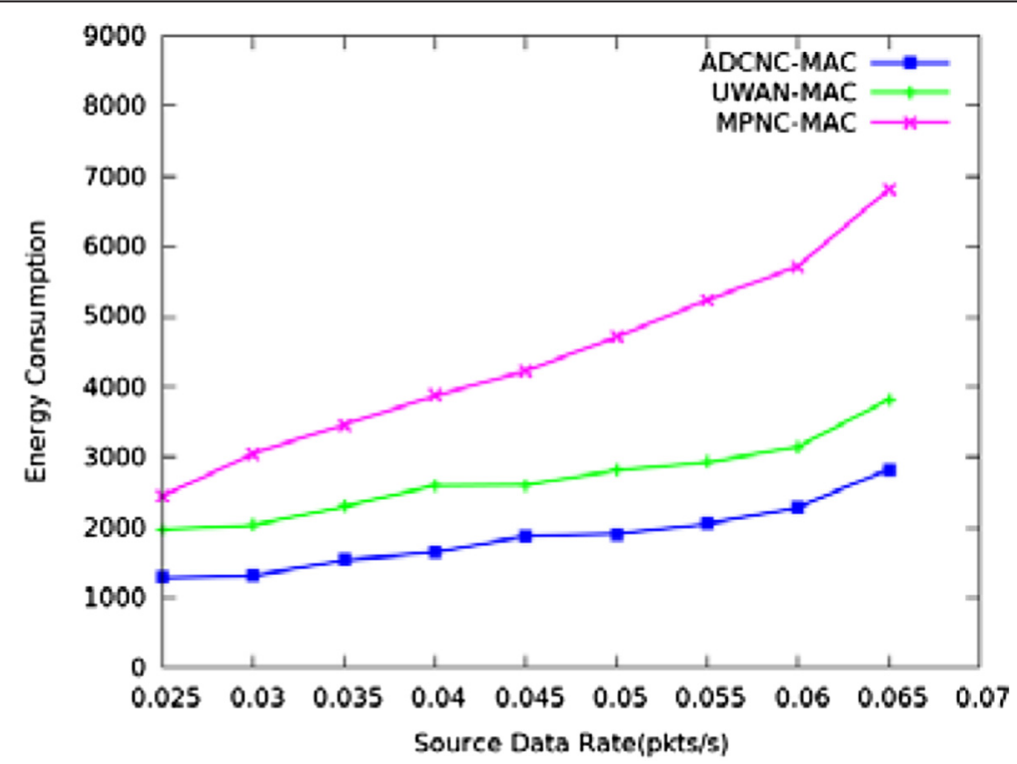

Fig. 12 The relationship between energy consumption and source data rate of protocols 


\section{Competing interests}

The authors declare that they have no competing interests.

\section{Author details}

${ }^{1}$ College of Computer Science and Technology, Harbin Engineering University, Harbin 150001, China. ${ }^{2}$ College of Shipbuilding Engineering, Harbin Engineering University, Harbin 150001, China.

Received: 5 February 2015 Accepted: 7 August 2015

Published online: 22 August 2015

\section{References}

1. G Acar, A Adams, ACMENet: an underwater acoustic sensor network protocol for real-time environmental monitoring in coastal areas. Radar, Sonar and Navigation, IEE Proc. 153(4), 365-380 (2006)

2. L Freitag and M Stojanovic, "Acoustic communications for regional undersea observatories," Proceedings of Oceanology International, London, UK 2002

3. IF Akyildiz, D Pompili, T Melodia, Challenges for efficient communication in underwater acoustic sensor networks. SIGBED Rev. 1(2), 3-8 (2004)

4. JH Cui, J Kong, M Gerla, and S Zhou, "Challenges: building scalable mobile underwater wireless sensor networks for aquatic applications," IEEE Network, Special Issue on Wireless Sensor Networking (NY, USA, 2006) pp. 12-18

5. J Heidemann, W Ye, J Wills, A Syed, and Y Li, "Research challenges and applications for underwater sensor networking," (IEEE WCNC, NY, USA, 2006)

6. L Freitag, M Grund, S Singh, J Partan, P Koski and K Ball, The WHOI Micro-Modem: an acoustic communications and navigation system for multiple platforms. (In IEEE Oceans, Washington, DC, Sep. 2005)

7. Y Sun, O Gurewitz, DB Johnson, Ri-mac: a receiver initiated asynchronous duty cycle mac protocol for dynamic traffic loads in wireless sensor networks, in SenSys'08: proceedings of the 6th ACM conference on Embedded network sensor systems (ACM, New York, NY, USA, 2008), pp. 1-14

8. Joseph Polastre, Jason Hill, and David Culler. Versatile low power media access for wireless sensor networks. In Proceedings of the Second International Conference On Embedded Networked Sensor Systems (SenSys, NY, USA 2004), pages 95-107

9. W Ye, J Heidemann, D Estrin, Medium access control with coordinated adaptive sleeping for wireless sensor networks. IEEE/ACM Trans Netw 12(3), 493-506 (2004)

10. M Obaidur Rahman, A Muhammad Mahbub, M Muhammad Mostafa, S Choong, nW-MAC: multiple wake-up provisioning in asynchronously scheduled duty cycle MAC protocol for wireless sensor networks. Ann Telecommun. 66, 567-582 (2011)

11. MK Park, V Rodoplu, UWAN-MAC: an energy-efficient MAC protocol for underwater acoustic wireless sensor networks. (IEEE Journal of Oceanic Engineering, Washington DC, VOL. 32, NO. 3, JULY 2007)

12. R Ahlswede, N Cai, SYR Li, R Yeung, Network information flow. IEEE Trans Inf Theory. 46(4), 1204-1216 (2000)

13. W Ye, J Heidemann, D Estrin, Medium access control with coordinated adaptive sleeping for wireless sensor networks. IEEE/ACM Trans Networking. 12(3), 493-506 (2004)

14. J Rice, B Creber, C Fletcher, P Baxley, K Rogers, K McDonald, D Rees, M Wolf, S Merriam, R Mehio, J Proakis, K Scussel, D Porta, J Baker, J Hardiman, D Green, Evolution of Seaweb underwater acoustic networking. in Proc MTS/IEEE OCEANS. 3, 2007-2017 (2000)

15. A Benson, J Proakis, M Stojanovic, Towards robust adaptive acoustic communications. in Proc MTS/IEEE OCEANS. 2, 1243-1284 (2000)

16. G Xie, J Gibson, A networking protocol for underwater acoustic networks[R] (Naval Postgrad, School Monterey CA Dept Of Computer Science, 2000)

17. G Zheng, B Wang, $P$ Xie, W Zeng, JH Cui, Efficient error recovery with network coding in underwater sensor networks. Ad Hoc Netw. 7(4), 791-802 (2009)

18. S Cai, Z Gao, DS Yang, A network coding based protocol for reliable data transfer in underwater acoustic sensor. Ad Hoc Netw. 11, 1603-1609 (2013)

19. S Katti, H Rahul, W Hu, D Katabi, M Medard, J Crowcroft, XORs in the air: practical wireless network coding. IEEE Trans Networking. 16, 497-510 (2008)

20. Z Zhou, J Cui, S Zhou, "Localization for Large-Scale Underwater Sensor Networks [C]." Proceedings of IFIP Networking (Springer, Atlanta, Georgia, USA, 2007)

21. The Network Simulator - ns-3, <http://www.nsnam.org>. 2014-06-15

\section{Submit your manuscript to a SpringerOpen ${ }^{\odot}$ journal and benefit from:}

- Convenient online submission

Rigorous peer review

- Immediate publication on acceptance

- Open access: articles freely available online

- High visibility within the field

- Retaining the copyright to your article

Submit your next manuscript at springeropen.com 